\title{
SEBASTIAN ZIÓŁKOWSKI
}

Université de Gdańsk

\section{La pièce climatique d'Henri-René Lenormand ou un désarroi ontologique}

e théâtre d'Henri-René Lenormand (1882-1951) et onirique semble peu compatible avec l'esprit cartésien français et suscite toujours des analyses originales. Grâce aux travaux de Maurice Gravier et, plus récemment, ceux de Tomasz Kaczmarek, elle a été examinéeà l'aune de l'expressionnisme dramatique européen ${ }^{1}$. La production dramatique de Lenormand fait fi de l'entendement traditionnel des catégories spatio-temporelles, instaure des ressassements identitaires complexes et véhicule un message foncièrement pessimiste sur le destin humain. Mais elle frappe avant tout par la pluridimensionnalité des motifs ressassés (dédoublement du personnage, apparition de personnages-satellites, spiritisme, climat désagrégeant l'individu, etc.). Et c'est justement cette diversité des prismes adoptés par le dramaturge qui favorise toujours de nouvelles approches analytiques.

L'un des motifs les plus emblématiques de cette œuvre inclassable est l'influence prépondérante du climat sur l'individu. Dans quelques-uns des drames lenormandiens, le climat est pour ainsi dire l'ossature de la construction dramatique, l'élément conditionnant le monde pré-

\footnotetext{
${ }^{1} \mathrm{Cf}$. T. Kaczmarek, "Henri-René Lenormand et l'expressionnisme dramatique ", [dans :] Acta Universitatis Lodziensis. Folia Litteraria Romanica, 2008, n 5. Cf. également M. Gravier " L'expressionnisme dramatique en France entre les deux guerres ", [dans :] L'expressionnisme dans le théâtre européen, Paris, CNRS, 1971.
} 
senté et influant sur les agissements des protagonistes. Certaines pièces de Lenormand, dont il sera question dans le présent article, peuvent être qualifiées de climatiques car il s'agit de drames où les personnages sont exposés aux épreuves d'un climat implacable et anxiogène, un climat qui les désagrège et éveille en eux des passions (auto-)destructrices. Un climat qui est donc loin d'être un décor secondaire du drame.

L'un des phénomènes que l'on retrouvera de manière récurrente à travers le théâtre de Lenormand est le brouillard, élément majeur participant de la désobjectivisation de l'univers présenté. L'illustration la plus parlante nous en est donnée dans Le temps est un songe (1921) ainsi que dans L'Amour magicien (1929). Le brouillard semble en effet propice à l'émergence des soupçons dans un univers à mi-chemin entre le réel et la fantasmagorie. La tombée des brouillards est un oiseau de mauvais augure : elle présage un renversement tragique de situation ou advient avec le malheur. Cependant, c'est aussi le climat tropical qui met les personnages dans des états d'âme des plus éprouvants. Le climat tropical rend plus indépassable et plus palpable le tragique des personnages lenormandiens, il le matérialise et exige de l'homme la plus grande endurance physique et psychologique.

Notons que Lenormand ébauche son premier drame climatique en 1910. II s'agit de Terres chaudes, pièce constituant sa première tentative de rendre l'influence dévastatrice du climat africain sur les Européens et annonçantsa poétique onirique concentrée sur la noirceur de l'âme humaine. Remarquonstoutefois qu'en écrivant Terres chaudes, le dramaturge ne connaissait pas l'Afrique par le biais de ses propres expériences si bien que la représentation qu'il endonne dans sa pièce est le fruit de son imagination, la confluence de visions fantasmagoriques, de lieux communs et d'invention dramatique. 
Deux phénomènes climatiques apparaissant dans les pièces " exotiques » de Lenormand seront à considérer : une chaleur insupportable et persistante (À l'ombre du $m a l)$ ainsi que le vent tropical engendrant des actes cruels (Le Simoun). La chaleur et les rudes épreuves du climat tropical favoriseront chez les personnages des réactions de violence et de démesure et serontfavorablesà l'action. En revanche, le brouillard et l'humidité du climat auront comme corollaire un état d'inertie et une stagnation destructrice.

Notons au passage la veine autobiographique de l'œuvre lenormandienne. En effet, l'auteurdu Temps est un songe était convaincu de l'influence du climat sur le bien-être psychique. Pareillement à August Strindberg, Lenormand considérait le brouillard comme un phénomène déclencheur de forces inapprivoisées du psychisme humain. Dans son œuvre, « le dramaturge transpose [...] l'influence du climat qui conditionnait sa propre disposition mentale. On sait que Lenormand était mal à l'aise quand il faisait du brouillard [...]. La chaleur tropicale tracassait également son psychisme au même point que ses personnages ${ }^{2}$. Aussi les personnages deviennent-ils, au dire de Lenormand, "le jouet des forces naturelles ${ }^{3}$, car, poursuit le dramaturge, " cette notion du drame, intégrant au domaine du théâtre les forces naturelles et surnaturelles [I]e séduisait $n^{4}$.

Dans le présent article, nous tâcheronsd'indiquer dans quelle mesure les enjeux se rapportant au climat décelables dans l'œuvre de Lenormand conditionnent la portée existentielle et ontologique des pièces que nous analyserons. Nous choisirons, comme nous l'avons signalé supra, deux axes de réflexion. II s'agira respectivement de l'iner-

\footnotetext{
${ }^{2}$ T. Kaczmarek, " Henri-René Lenormand et l'expressionnisme dramatique ", op. cit., p. 50.

3 H.-R. Lenormand, Les confessions d'un auteur dramatique, Paris, Albin Michel, 1949, vol. 1, p. 12.

4 Ibidem, p. 13.
} 
tie devant le malheur (à travers le motif du brouillard notamment et celui de l'humidité du climat) et du déchaînement de passions meurtrières (pièces " exotiques » dépeignant un climat violent : une chaleur insupportable et le vent africain, le simoun).

Brouillard ou inertie devant le malheur : L'Amour magicien (1929)5

La première des pièces climatiques à laquelle nous consacrerons quelques lignes est L'Amour magicien. II s'agit d'un drame onirique en trois actes et six tableaux rompant avec toute poétique réaliste et nous mettant aux prises avec un individu victime de ses passions inavouées.

Dans la pièce en question, Lenormand conçoit la mystérieuse histoire du suicide d'une femme : un soir de brouillard, Berthe Carolles est sortie de chez elle pour faire, paraît-il, sa promenade quotidienne au bord de la mer et n'est jamais revenue depuis. Tout le monde pense subitement à une noyade. Mais personne n'ose envisager explicitement un autre scénario, plus probable : le suicide. L'accident semble en effet peu plausible vu que l'héroïne connaissait les lieux et que les conditions météorologiques n'étaient guère anormales le jour de sa disparition. Néanmoins, le mystère persiste, car son corps demeure introuvable. Pour asseoir, semble-t-il, ce sentiment d'énigme indépassable, les brouillardssont évoqués déjà pour décrire les habitudes de la disparue et on apprend que Madame Carolles " aimait s'exposer aux brouillards, aux embruns " $(A M, 24-25)$. On a affaire là à un élément ressassé obsessionnellement. Dans les didascalies du troisième tableau, on apprend que l'action se déroule dans « un calvaire sur la lande, par un paisible soir [et qu'il fait

${ }^{5}$ Cf. H.-R. Lenormand, L'amour magicien, [dans :] Idem, L'amour magicien, Innocente, Théâtre complet 6, Paris, G. Crès et Cle, 1929. Les citations provenant de cette œuvre seront marquées à l'aide de l'abréviation $A M$, la pagination après le signe abréviatif. 
du] brouillard sur la mer " $(A M, 46)$. Et plus loin Albert de constater : "Quel brouillard sur la mer! Toute l'île est ceinturée de brume ! On dirait une écharpe de lait » (AM, 47). À un autre moment encore, Albert et Edouard sont obligés d'écourter leur promenade et de rentrer aussitôt, sinon ils seront surpris par la " nuit blanche " $(A M, 57)$.

Albert, époux de l'héroïne, se remémore le soir du drame :

Vers 9 heures, la brume est tombée avec la nuit et j'ai commencé à m'inquiéter. Je suis parti à sa recherche [...]. Les pierres où nous avions coutume de nous asseoir étaient humides... Oui, une lame plus forte a pu l'emporter... Mais tout le promontoire était moite de brouillards et d'embruns... $(A M, 11-12)$

L'ambiance de la pièce est ineffable : les brouillards et l'omniprésente humidité désobjectivisent la scène et amplifient le sentiment d'une vérité échappant à Albert Carolles $^{6}$. Cette dernière demeure aussi insaisissable que les embruns d'une soirée brumeuse à la côte bretonne, le paysage dans lequel Lenormand situe l'action du drame.

Les brumes font en sorte que l'inquiétude ne cesse de hanter le héros. II ressasse continuellement le souvenir de la disparition de Berthe : " Je ne peux pas encore voir tomber la nuit dans cette pièce. Voici les brumes qui montent de l'ouest [...]. Elle est sortie dans un moment pareil " $(A M, 17)$. Le brouillard attise l'inquiétude et évoque des souvenirs tourmentant Albert.

De par une profonde déréalisation de l'univers de la pièce, le dramaturge va jusqu'à user du motif de la migration de l'âme, motif chéri de Lenormand et pivot de la pièce. L'ambiance anxiogène va crescendo. Berthe communique avec son mari par l'intermédiaire de Beatrice qui devient un médium. La jeune fille d'avouer : « Je crois et je croirai qu'elles [les âmes des défunts] circulent au fond

${ }^{6}$ Berthe Carolles menait une double vie : elle trompait son mari. Chose encore plus inavouable, son amant était le beau-frère d'Arthur, époux de Berthe. 
des bois de chênes, se reposent au bord des étangs et voyagent avec le brouillard " $(A M, 97)$.

Berthe hante également les rêves de son mari désespéré. Aussi la représentation de sa femme entrevue dans les rêves est-elle pour Albert " une espèce de brouillard sonore " $(A M, 107)$. Le brouillard acquiert ainsi une dimension métaphysique et métaphorique. Voici Albert de déclarer : " J'ai cru un moment que j'allais réussir à crever les cloisons de brouillards et d'incertitude entre lesquelles se débat l'humanité... " $(A M, 81)$, le brouillard étant la métaphore de ce qu'il y a de plus insaisissable dans l'existence.

L'Amour magicien est donc, on l'a vu, une pièce dont l'ambiance est conditionnée par les phénomènes météorologiques. Brouillard, humidité, tempête : autant de motifs créant cet univers où l'individu se désagrège et reste désarmé devant le tragique de son sort.

\section{Le temps est un songe (1921) 7}

L'action de la pièce est située en Hollande, "de nos jours » (TS, 148). Romée vient rencontrer, après une longue séparation, son fiancé Nico, venu des Indes pour l'épouser. En se rendant dans la maison familiale de ce dernier, elle longe un étang et aperçoit, à travers le brouillard, la tête de Nico affleurer à la surface de l'eau. Une ambiance d'incertitude s'instaure ainsi car notre héroïne ne sait plus si la scène entrevue est réelle ou bien si cette vision découle d'un leurre phénoménologique, d'une hallucination macabre.

Pourtant le lecteur s'aperçoit vite que la scène est en train de se matérialiser tout au long du drame. Nico, qui est revenu des Indes, semble plongé dans une dépression-

\footnotetext{
7 H.-R. Lenormand, Le temps est un songe, [dans :] Idem, Les Ratés, Le Temps est un songe, Théâtre complet 1, Paris, Albin Michel, 1921. Les citations provenant de cette œuvre seront marquées à l'aide de l'abréviation $T S$, la pagination après le signe abréviatif.
} 
profonde et est envahi par des doutes sur la réalité même du monde. Il descend cette pente de la dépossession de soiet s'achemine vers le suicide, devant survenir, comme relevant d'une malédiction, à la fin de la pièce. Lenormand réalise ainsi son idée novatrice de continuum de temps selon laquelle passé, présent et avenir sont des notions temporelles périmées et ne font plus qu'un.

Dans Le temps est un songe, Lenormand, comme nous l'avons observé supra à propos de L'Amour magicien, esquisse un monde dominé par l'humidité où il ne fait jamais soleil : "Onze heures du matin et il fait nuit... » (TS, 175) déplore Nico. Car même s'il est onze heures du matin, " les brouillards collent si obstinément à la vitre qu'il règne une demi-nuit dans la pièce " $(T S, 172)$. Ces phénomènes, nous l'avons signalé à propos de L'Amour magicien, participent de la dématérialisation de l'univers de la pièce. Toutefois, relevons une ambiguïté significative concernant I'incessante interpénétration du monde réel et imaginaire. De fait, les événements survenant dans le drame sont la confluence du vrai et de l'illusion. Tantôt on a l'impression que les brouillards sont dans Le temps un phénomène naturel et point irréaliste, dus à l'humidité du climat, tantôt ils ne semblent découler que d'une vision hallucinatoire survenue à un personnage en proie à la désagrégation de l'appareil psychique : "Tu as parlé du brouillard. II n'a pas fait de brouillard »(TS, 160), déclare Riemke, inquiétée par l'improbable témoignage de son amie.

Cette oscillation entre l'évidence et l'illusion commence à gagner les autres personnages du drame. Mais c'est Nico qui souffre le plus à cause de ce climat délétère. II s'en plaint ainsi en répondant à la question de Romée voulant découvrir la cause de sa profonde apathie : " [...] ces vapeurs grises qui passent, qui passent pendant des semaines... Cette pluie qui est encore de la brume... Cette brume qui est déjà de la pluie... cela me désagrège " (TS, 174). Ceci n'est pas surprenant vu qu'il a passé sa jeunesse sous les chaleurs des Indes, le contraste et le chan- 
gement climatiques étant flagrants. D'ailleurs, les Indes ont profondément marqué notre héros. II y a découvert des théories mystiques orientales prônant la relativité du savoir de l'homme sur l'existence ainsi que suggérant l'aspect illusoire des catégorisations spatio-temporelles traditionnelles (TS, 194-199).

Au début de son séjour en Hollande, Nico est tellement irrité par l'aspect pourri et humide du paysage qu'il ambitionne de changer de fond en comble son environnement immédiat : il fait faire des travaux d'aménagement et envisage des réparations autour de la demeure familiale. Pourtant il ne tarde pas à s'enferrer dans une sorte de transe teintée de désappointement et d'inertie et il ne trouve plus la moindre envie de se révolter contre ses états d'âme.

Son état mental empire de jour en jour si bien que le doute, voire la dénégation, semblent désormais ses uniques points de repère dans un monde devenant de plus en plus vague. Nico de définir en ces termes le mal qui le ronge :

C'est le doute... le doute sur tout... sur la vie... sur les choses, sur moimême. Quand j'étais enfant, je m'imaginais que mon existence était une illusion... Je ne trouvais pas, dans mes sensations, de preuves suffisantes pour croire que j'étais réellement en vie [...]. Un savant à qui je fis part de cette idée, me répondit qu'il la comprenait, qu'il ne la trouvait pas absurde... C'est cette année-là que j'ai voulu me tuer. (TS, 195-196)

De fait, ses doutes ne le quitteront plus : ayant perdu tout contact avec le monde "réel ", il s'achemine vers l'étang bordant la route près de la maison pour mettre fin à ses jours. Ainsi la prémonition de Romée se trouve-t-elle réalisée et l'impression que c'est justement le climat humide de Hollande qui a tué Nico semble pertinente.

En dépeignant une vision aussi pessimiste du monde, notre dramaturge se révèle le continuateur de la philosophie de Nietzche. L'auteur du Temps est un songe instaure un univers dont Dieu se retira définitivement et où l'individu est condamné à une stagnation meurtrière. Le monde dépeint dans ses pièces fait aussi penser à la philosophie 
schopenhauerienne et il ne semble plus qu'une représentation mentale ou imaginative, si ce n'est une chimère, vision tellement chère à l'auteur du Monde comme volonté et comme représentation. D'autre part, Lenormand s'inspirait, tout comme Strinberg, du bouddhisme et du mysticisme oriental. Ainsi, " écrivant les premières scènes du drame [Le temps est un songe] sous l'emprise de Strindberg, le dramaturge semble privilégier la thèse selon laquelle l'univers n'est qu'une apparence (= une farce ou un néant relatif) ${ }^{8}$. Mais le pessimisme lenormandien acquiert une dimension toute particulière car il est suggéré, nous l'avons observé, par l'exploitation du motif de l'influence du climat sur l'attitude existentielle du personnage.

Climat tropical : déchaînement des passions et règne du mal

Aux dires de Tomasz Kaczmarek, Le Simoun ${ }^{9}$, pièce répartie en tableaux, est la plus représentative du théâtre climatique lenormandien ${ }^{10}$. L'importance du climat est soulignée au niveau du titre, le simoun étant un vent tropical entraînant des orages de sable, un vent " dépersonnalis[ant] les Européens ${ }^{11}$. En choisissant le simoun comme motif principal de la pièce, Lenormand s'inspire de la dramaturgie strindbergienne. Le Suédois explora le même motif en 1889 dans sa pièce au titre presque identique (Simoun $)^{12}$ et insista sur l'influence néfaste du vent tropical sur la psyché des Européens. On n'a qu'à évoquer les

${ }^{8}$ T. Kaczmarek, " Henri-René Lenormand et l'expressionnisme dramatique ", op. cit., p. 86.

${ }^{9}$ H.-R. Lenormand, Le Simoun, [dans :] Idem, Le simoun, Le mangeur de rêves, Théâtre complet 2 , Paris, G. Crès et $\mathrm{Cl}^{\mathrm{l}}$, 1922. Les citations provenant de cette œuvre seront marquées à l'aide de l'abréviation $S$, la pagination après le signe abréviatif.

$10 \mathrm{~T}$. Kaczmarek, "Henri-René Lenormand et l'expressionnisme dramatique », op. cit., p. 50.

11 Ibidem, p. 51.

${ }^{12}$ Cf. A. Strinberg, Simoun, A. Lindkvist (trad.), [dans :] Idem, Le théâtre complet 2, Paris, L'Arche, 1982. 
propos de l'auteur de Mademoiselle Julie qui considérait que le simoun " dessèche le cerveau des blancs, et... ils ont des visions terribles qui leur rendent la vie détestable et les font s'élancer vers le grand espace inconnu $»^{13}$.

Dans Le Simoun, nous découvrons l'existence d'un certain Laurency, un fonctionnaire colonial français vivant dans le M'Zab avec sa compagne Aïescha. On apprend qu'après la mort de sa femme restée en France, sa fille Clotildeva le rejoindre, à la plus grande indignation d'Aïescha. Dans les didascalies du premier tableau de la pièce on lit que « la toile se lève sur une après-midi de grande chaleur et de torpeur silencieuse » $(S, 6)$. Quelques pages plus loin, le receveur se plaint de la fièvre qu'il ne peut nullement maîtriser $(S, 27)$. Des manifestations somatiques du climat rude ne cessent de rendre l'existence des Européens pénible.

Les remarques concernant le climat se font de plus en plus récurrentes tout en gagnant en intensité descriptive : "Même lieu, le soir du même jour. Un violent crépuscule de pourpre teint le ciel et la chebka » $(S, 32)$. L'impact du climat implacable régnant dans le pays transparaît dans de nombreuses phrases prononcées par Laurency. Ainsi le héros de faire part de ses regrets : " Si je ne m'avais pas laissé manger par ce traître de pays » $(S, 38)$ ou de ses expériences pénibles : "J'étais tombé près d’un marais, foudroyé par une fièvre pernicieuse » $(S, 46)$. D'autres indications climatiques suivent. Ainsi L'Agha avertit-il Laurency qu' « en juillet, il n'y a pas de bon voyage à travers la chebka : toujours les tables de pierre, le ciel blanc et le vent de feu » et Laurency ajoute qu' « [il] crain[t] pour [sa] fille le soleil... la grande chaleur » particulièrement pernicieux car « le climat est mauvais » $(S, 51)$.

13 Propos de Strinberg cités d'après : T. Kaczmarek, « Henri-René Lenormand et l'expressionnisme dramatique », op. cit., p. 51. 
Les effets des grandes chaleurs agissent sur les sentiments des personnages et sont en quelque sorte propices à l'éveil d'instincts refoulés. Ces derniers sont à même de tarir la lucidité de la raison cartésienne censée propre à l'esprit rigoureux des colonisateurs. Dans Le Simoun, Laurency s'éprend ainsi d'une passion adultère de sa propre fille. En lisant la pièce, on a l'impression que c'est justement sous l'effet du climat éprouvant qu'il succombe à des rêves coupables déclenchant pour ainsi dire le conflit dramatique. D’ailleurs, comme le remarque Aïescha, " il n'y a guère d'Européens qui résistent à de pareils étés " $(S, 91)$. Clotilde, la fille de Laurency, tombe amoureuse de Giaour, musulman, fils de l'Agha des Laarba, ennemi juré de Laurency. Leurs conversations reflètent, elles aussi, la violence du climat. Giaour d'affirmer : "On étouffera partout cette nuit » $(S, 124-125)$.

Les allusions au climat éprouvant ne cessent d'affluer au niveau des passages descriptifs ouà travers les échanges entre protagonistes et mettent en valeur le tragique de l'individu. La fusion de l'intériorité de la vie des protagonistes et de l'extériorité d'un monde au climat des plus extrêmes semble ainsi particulièrement soulignée. Dans cette perspective, le climat paraît être sinon l'unique force agissante de la pièce, du moins un personnage à part entière ayant le plus d'impact sur le déroulement de l'intrigue.

\section{À l'ombre du mal (1925) ${ }^{14}$}

Il s'agit d'une autre pièce vilipendant les iniquités de l'empire colonial français. Une fois encore, notre dramaturge fait coïncider les considérations d'ordre socio-politique avec des enjeux propres au climat. L'action de la

${ }^{14} \mathrm{H}$.-R. Lenormand, À l'ombre du mal, [dans :] Idem, L'Homme et ses fantômes, À l'Ombre du mal, Théâtre complet 4, Paris, G. Crès et Cle, 1925. Les citations provenant de cette œuvre seront marquées à l'aide de l'abréviation $O M$, la pagination après le signe abréviatif. 
pièce se déroule vers 1905, dans la résidence de Kadésio, en Afrique équatoriale française $(O M, 124)$. Elle nous met en scène notamment quatre protagonistes : Le Cormier, Madame Cormier (son épouse), Rougé, résident français de Kadésio, ainsi que Préfailles, administrateur colonial. Monsieur Cormier, subordonné de Rougé, s'est installé à Kadésio il y a moins d'un an, mais peine à s'accommoder au climat et ne supporte pas l'ambiance délétère régnant dans la communauté marquée par nombre de conflits (entre clans autochtones ainsi qu'entre autochtones et colonisateurs).

La pièce est bâtie sur un axe bipolaire : Cormier et sa femme se montrent prévenants pour les autochtones; a contrario, Rougédemeure beaucoup plus intransigeant : son attitude envers le peuple gouverné fait preuve de racisme et de xénophobie. Il est l'emblème caricatural du colonisateur français.

Préfailles, quant à lui, semble beaucoup plus modéré dans ses jugements que Rougé; ce n'est que plus tard qu'on découvre que, par le passé, il a détruit la vie de Rougé et qu'il lui a occasionné des souffrances (OM, 197205). Une fois encore, on assiste donc au spectacle d'un mal omniprésent rongeant l'être humain et envenimant ses relations avec autrui. Et il semble de nouveau que tout cela soit motivé par l'implacable climat propice au déchaînement de pulsions destructrices. Soit ainsi dit en passant, Madame Cormier est assassinée par les autochtones qui auraient, avec cet acte de barbarie, imité l'agissement et la loi des Blancs.

Les indications ayant trait aux phénomènes climatiques percent déjà dans les didascalies précédant le premier acte du drame. On lit ainsi que " la lumière implacable [...] embrase l'espace défriché et prend d'assaut la véranda $[. .$.$] " (O M, 126)$ si bien qu'on se doit d'inventer des dispositifs pour " se défendre de la lumière " $(O M$, 126-127). Et un peu plus loin suivent des précisions se rapportant au climat qui accompagnent les indications 
temporelles, bien marquées dans À l'ombre du mal (ce qui est un procédé inhabituel dans la dramaturgie lenormandienne faisant du brouillage de tout repère de temps et d'espace son fer de lance dans la lutte contre le réalisme et le naturalisme au théâtre) : " II est trois heures d'une brûlante après-midi de la saison des pluies » $(O M, 126)$.

Rougé a pris en horreur le folklore des autochtones. Le seul fait de les voir danser, se réjouir, le met dans tous ses états. Le fait qu'ils en soient capables par des chaleurs intenables le met davantage hors de lui. Le voici de constater : " 43 degrés ! Et ça chante ! Et ça danse ! Et ça ne crève même pas d'un coup de soleil ! " $(O M, 142)$. Il se plaint constamment des tristes effets que l'avènement des phénomènes météorologiques propres à la région a sinon sur sa santé, du moins sur son bien-être général : " C'est cette tornade qui approche... Je n'ai guère de répit à espérer, d'ici la saison sèche » $(O M, 187)$.

Il semble toutefois que la meilleure définition de l'empreinte que laisse l'Afrique sur l'individu ait été proposée par Préfailles élucidant les motivations de sa conduite répréhensible envers Rougé à l'époque où ils vivaient tous les deux en Côte d'Ivoire (OM, 197-200). Préfailles s'efforce de contextualiser les exactions commises et constate que la cause possible de sa férocité, c'était

l'Afrique, peut-être [...]. Cette coulée de jours vides, [...] embrasés d'une même flamme [...]. Au milieu de ce désert, un vent de rage vous tord brusquement... Faire souffrir. II faut faire souffrir ! On ne raisonne plus [...]. $(O M, 224)$

Sous le climat tropical, l'esprit rationnel abandonne l'homme, victime des instincts les plus vils, un monstre.

Le climat joue dans toutes les pièces analysées un rôle prépondérant : il dicte les agissements des personnages, déréalise l'univers présenté et éveilleles forces ignorées de l'inconscient. L'instauration d'une relation de cause à effet entre le climat rude et le tragique existentiel constitue un procédé original et fait de Lenormand l'un des 
dramaturges les plus novateurs de l'entre-deux-guerres en France et un digne successeur d'August Strindberg. Par ailleurs, les conclusions de l'étude du théâtre lenormandien concordent avec les analyses contemporaines de l'influence du climat sur le psychisme humain selon lesquelles « le milieu au sens de l'entourage humain et physique joue un rôle important dans la formation de l'être humain. II le modèle littéralement ${ }^{15}$. Et, à l'heure actuelle, "le schéma de la plupart des chercheurs est [toujours] un schéma naturaliste qui peut se résumer dans la formule : la nature [et donc le climat] fabrique l'homme ${ }^{16}$. On saisit mieux ainsi que la peinture de la psychétracassée par un climat pernicieux n'est pas un motif sur anné, mais correspond aux acquis de la réflexion contemporaine sur la question.

Date de réception de l'article: 18.02.2019. Date d'acceptation de l'article : 24.03.2019.

$15 \mathrm{M}$. Lobrot, Le psychisme en mouvement : Tome 3, L'écologie humaine, Saint-Denis, Publibook, 2017, p. 12.

16 Ibidem, p. 13. 


\section{bibliographie}

Gravier M., "L'expressionnisme dramatique en France entre les deux guerres ", [dans :] L'expressionnisme dans le théâtre européen, Paris, CNRS, 1971.

Kaczmarek T., « Entre le temps de Kant et le temps d'Einstein, ou requête pour un texte expressionniste français " [dans :] Études romanes de Brno, 2010, n०31.

Kaczmarek T., " Henri-René Lenormand et l'expressionnisme dramatique ", [dans :] Acta Universitatis Lodziensis. Folia Litteraria Romanica, 2008, n० 5. Kaczmarek T., Le personnage dans le drame français face à la tradition de l'expressionnisme européen, Łódź, Wydawnictwo Ut, 2010.

Lech-Galland C., La réception du théâtre français en Allemagne (19181933), Paris, Honoré Champion, 1998.

Lenormand H.-R., À l'ombre du mal, [dans :] Idem, L'Homme et ses fantômes, À l'Ombre du mal. Théâtre complet 4, Paris, G. Crès et Cie, 1925. Lenormand H.-R., L'amour magicien, [dans :] Idem, L'amour magicien, Innocente, Théâtre complet 6, Paris, G. Crès et Cie, 1929.

Lenormand H.-R., Les confessions d'un auteur dramatique, Paris, Albin Michel, 1949, vol. 1.

Lenormand H.-R., Le Simoun, [dans :] Idem, Le simoun, Le mangeur de rêves, Théâtre complet 2, Paris, G. Crès et Cie, 1922.

Lenormand H.-R., Le temps est un songe, [dans :] Idem, Les Ratés, Le Temps est un songe, Théâtre complet 1, Paris, Albin Michel, 1921.

Lenormand H.-R., L'Homme et ses fantômes [dans :] Idem, L'Homme et ses fantômes, À l'Ombre du mal. Théâtre complet 4, Paris, G. Crès et Cie, 1925. Lobrot M., Le psychisme en mouvement : Tome 3, L'écologie humaine, SaintDenis, Publibook, 2017.

Morrison W. G., The treatment of the problem of evil in the theatre of Lenormand, Hamilton, Presses Universitaires d'Université McMaster, 1972.

Strinberg A., Simoun, A. Lindkvist (trad.), [dans :] Idem, Le théâtre complet 2, Paris, L'Arche, 1982.

\section{abstract}

\section{The Climatic Play by Henri-rené Lenormand or an Ontological Disarray}

The aim of this paper is to analyse the climatic plays by Henri-René Lenormand (1882-1951), and to show how difficult climatic conditions shown in his plays influence the behaviour and the attitude of the characters. In the paper different motifs are taken into consideration : fog (and, therefore, the humidity of the climate), the tropical climate in general and the tropical wind called lesimoun. In Lenormand's plays climate is not just a secondary motif, devoid of significance, but an element which conditions the atmosphere of those plays, engenders a deep melancholy or awakens hidden and repressed instincts of the characters. The climate emphasises thus a deep existential pessimism and the tragic of an individual lost in a hostile and incomprehensible world. 


\section{keywords}

Lenormand, climatic play, fog, tropical climate, tragic vision of the world

\section{mots-clés}

Lenormand, pièce climatique, brouillard, climat tropical, vision tragique du monde

\section{sebastian ziólkowski}

Diplômé en philologie romane (Université de Gdańsk). En 2017, il a soutenu le mémoire de licence portant le titre : Le tragique de l'individu dans l'œuvre romanesque de Louis-Ferdinand Céline. En juillet 2019, il a soutenu son mémoire de maîtrise intitulé Du pré-symbolisme à l'expressionnisme dramatique en France. II s'intéresse avant tout à la littérature du $\mathrm{XIX}$ et de la première moitié du XXe siècle et, d'une manière toute particulière, aux avant-gardes françaises du siècle écoulé.

ORCID : 0000-0001-8763-4951 